los colorantes más utilizado en el campo de la resturación textil.

En base a este grupo innovador de cibacron, los tres grandes bloques del programa han estado enfocados al desarrollo de una amplia gama de ensayos llevados a cabo con este grupo de colorantes sobre telas de procedencia orgánica. Estas pruebas nos sirvieron para familiarizarnos con ellos a la vez que para constatar mendiante nuestros propia experiencia las ventajas sobre los solofeniles.

Estos ensayos consistiron en realizar una serie de tinturas cambiando los parámetros principales de las mismas (material, proporción del agua, proporción de los colorantes, temperatura o tiempo), los prductos químicos necesarios para la ejecución de

la tintura y de los productos auxiliares necesarios para producir mejor la reacción del tinte.

Fundamentalmente el progreso y avance de este grupo de cibacron en el campo de los tintes estriba en su mejor solidez a la luz, a la humedad y al calor, así como la mejor miscibilidad entre ellos y su más fácil tinción por simplificación de su fórmula. A su vez los nuevos colorantes contienen menos substancias perjudiciales para el medioambiente cumpliendo por tanto una responsabilidad ecológica.

Con este seminario la empresa Ciba ha cumplido uno de los objetivos presentes en su filosofía que es la de equilibrar sus responsabilidades económicas con las sociales. La divulgación de sus productos a un reducido grupo de restauradores textiles así como el suministro gratuito a los mismos de los colorantes utilizados son tareas que ponen de manifiesto el prestigio de esta entidad.

\section{ARQUEOLOGÍA Y CIUDAD}

El Instituto de Conservación y Restauración de Bienes Culturales ha impartido en Madrid durante los días 23, 24 y 25 de Abril un curso sobre arqueología urbana. En este caso, la ciudad ha servido de marco para expresar los problemas de la arqueología.

En la primera de las jornadas se puso especial énfasis en el soporte legal. La ponencia titulada, "Instrumentos legales de interven- ción y protección" dió pie a un amplio debate. En dicha ponencia, Concepción Martín ( ICRBC) y Félix Benito (ICRBC), hicieron un repaso de las distintas normativas existentes en el territorio español, tanto de la estatal como de las comunidades autónomas. Así mismo, se discutió sobre las normas de planeamiento, y entre otras cosas se planteó la necesidad de la presencia de un arqueólogo a la hora de elaborar las normas de planeamiento por parte de los ayuntamientos.

En un plano más práctico, en el segundo día y parte del primero, ponentes procedentes de distintas comunidades autónomas mostraron distintas experiencias. Ignacio Rodríguez Temiño (Junta de Andalucía) explicó el proyecto de arqueología urbana de Andalucía, Ricardo Mar de Tarragona (Universidad de Tarragona) hizo un repaso a la historia de la arqueología de la ciudad desde la creación del TEDA hasta la actualidad, momento en que la Universidad se ha hecho cargo de la gestión de la arqueología en el municipio. Arqueólogos de la Comunidad de Madrid definieron el complicado y polémico caso de la excavación de la Plaza de Oriente. Por su parte Sebastián Ramallo (Universidad de Murcia) presentó el largo proceso seguido hasta la consecución de la excavación del Teatro Romano de Cartagena. Linarejos Cruz (ICRBC) mostró, dentro de Europa, el modélico caso de la ciudad de Estrasburgo.

En todos los casos, además de los problemas que conlleva una excavación urbana, se planteaba la conservación de restos y para hablar de la protección física del patrimonio arqueológico urbano estuvieron Soledad Ruiz (ICRBC) y María Luisa González (Museo de Zaragoza), que dieron unas normas básicas de protección de los restos arqueológicos, además de reivindicar la presencia de la figura de un restaurador en cualquier intervención arqueológica.

Haciendo un paréntesis en lo puramente arqueológico, Manuel Manzano Monís (ICRBC) realizó un bello y profundo ensayo sobre la ciudad y sus habitantes.

Como punto final de las ponencias, Gonzalo Ruiz Zapatero (Universidad Complutense) habló de la rentabilidad social y científica de la arqueología urbana, mostrando algunos ejemplos de como acercar a la población los descubrimientos realizados por los arqueólogos y que forman parte su propia cultura.
María Dolores Fernández Posse, coordinadora del curso, lo clausuró haciendo un breve resumen de lo tratado durante los tres días y dando paso a una breve mesa redonda.

Pilar Mondéjar

Centro de Documentación del IAPH

\section{CONFERENCIA NACIONAL DE USUARIOS DE ESRI}

Durante los días 24, 25 y 26 de Abril tuvo lugar en el Hotel Mindanao de Madrid la Conferencia anual de usuarios de ESRI que celebraba su quinta edición. Además de la presentación en stands de los últimos productos informáticos ofertados por ESRI se presentaron ponencias referidas a diversas aplicaciones desarrolladas por usuarios de Arclnfo que se organizaron en áreas temáticas.

Invitados por los organizadores de la Conferencia asistimos el jueves día 25 a la presentación de experiencias en el área de medio ambiente, geología, agricultura, estudios forestales y ecología con el principal objetivo de conocer de primera mano el trabajo presentado por Carlos Almonacid del Servicio de Cartografía de la Universidad Autónoma de Madrid sobre el modelo de aplicación de los S.I.G. a la gestión del patrimonio arqueológico en la Comunidad de Madrid.

La aplicación, realizada mediante la colaboración de la Comunidad de Madrid, el Departamento de Prehistoria y Arqueología de la Universidad Autónoma de Madrid y el Servicio de Cartografía de la misma Universidad, se orientó específicamente hacia la Gestión del Patrimonio Arqueológico de Madrid marcándose los siguientes objetivos iniciales:

- Que respondiera a los requisitos alfanuméricos de cualquier Base de Datos.

- Que elaborara representaciones gráficas en soporte cartográfico.

- Que elaborara salidas cartográficas a consultas que incluyeran claves alfanuméricas y gráficas.

- Que poseyera un interfaz de usuario sencillo.

- Que tuviera posibilidades de crecimiento ampliándose a otras categorías de bienes y escalas cartográficas.

Las fases del proyecto se concretaron en un estudio metodológico preliminar de la infor- 
mación existente, en la configuración propia del sistema y en la implementación final. Así, se comenzó tomando los datos alfanuméricos referidos a los yacimientos arqueológicos, referenciándolos mediante un punto de coordenadas UTM a una base cartográfica a escala 1:25.000, cruzándose luego con otras capas de información referidas a la altimetría, límites municipales, red viaria, polígonos de edificabilidad, hidrografía, toponimia y áreas construidas.

La aplicación actualmente permite realizar consultas, composiciones, selecciones de yacimientos, salidas gráficas, generar áreas de influencia, introducir nuevos datos, actualizar información, etc.

Silvia Fernández Cacho Centro de Documentación del IAPH

\section{SECOND JOINT EUROPEAN CONFERENCE AND EXHIBITION ON GEOGRAPHICAL INFORMATION. $\checkmark$ CONGRESO DE LA ASOCIACIÓN ESPAÑOLA DE SISTEMAS DE INFORMACIÓN GEOGRÁFICA. Barcelona, Palacio de Congresos 27-29 de Mayo.}

Las Jornadas, organizadas por EUROGI, asociación europea de usuarios de Sistemas de Información Geográfica (SIG), se organizaron en siete bloques de comunicaciones: Tecnología, Desarrollo, Planeamiento, Gobiernos regionales y urbanos, Telecomunicaciones, Educación y Panorama español.

Junto a la valoración de los SIG como herramienta imprescindible en el apoyo a la toma de decisiones en el ámbito del planeamiento regional y la gestión de recursos, resaltar la comunicación presentada por $\mathrm{A}$. de la Torre Prados, bajo el título de 'Proyectos GIS en España: Estrategia y desarrollo en el marco de la política del Ministerio de Industria' . En ella se subrayó el esfuerzo adicional de normalización y cooperación con otras instituciones que conlleva el uso de los SIG, con el fin de evitar duplicidades tanto en la elaboración de las fuentes de información como en los resultados finales, de forma que se racionalicen las inversiones y los esfuerzos y se establezca un circuito fluido de circulación de la información.

Desde varias ponencias se incidió en la necesidad de orientar los SIG, junto a la agilización del ámbito de la gestión, a ofrecer a cualquier tipo de usuario el acceso cómodo a la información en función de sus necesidades específicas. En este sentido, junto a un necesario proceso de mejora de la relación (interfaz) con el usuario, analizada en trabajos como 'Tecnología Multimedia en los Sistemas de Información Municipales' o 'Un Sistema de Información Multimedia (M.I.S) al servicio de los ciudadanos', empieza a adquirir importancia el servicio remoto de la información, a través de los nuevos canales de información disponibles como Internet. En esta última línea se inscribió la comunicación 'Servidores de Geo-Datos en la WWW'.

La presentación de comunicaciones se completó con la asistencia de firmas comerciales que, en sus stands, ofrecieron una visión del amplio panorama de los SIG.

Las Actas de las Jornadas, recogidas en la publicación 'From Research to Application through Cooperation', se encuentran a disposición de los usuarios en la Biblioteca del IAPH.

\section{Ramón Pico Valimaña Centro de Documentación del IAPH}

\section{SECCIONES TÉCNICAS DEL CIETA}

Del 15 al 26 de Abril de 1996 se celebró en Lyon, Francia, la primera parte de la Sección Técnica organizada por el Centro Internacional para el Estudio de los Textiles Antiguos (CIETA).

Esta organización se funda en 1954 por iniciativa del Conservador del Museo de Mayence (RFA), M. Volbach; y su cede se establece en Lyon, en el Museo Histórico de Los Tejidos, propiedad de la Cámara de Comercio y de la Industria de Lyon.

Esta organización tiene como propósito promover un plan internacional que englobe toda acción que tienda a conocer mejor los tejidos antiguos, y coordinar las acciones llevadas a cabo por los diferentes países para realizar un inventario de los textiles.

Estos cometidos se ve reforzado por el vocabulario técnico textil, traducido a 7 lenguas (francés, italiano, español, lenguas escandinavas, alemán y portugués) y las Secciones Técnicas, en las que se forma a los profesionales en los métodos de análisis de los textiles.
Una Sección Técnica comprende dos cursos de dos semanas cada uno, separados por un intervalo de un año, realizándose durante los meses de Abril o Septiembre de cada año.

Las Secciones Técnicas se han venido realizado desde 1956, con la finalidad de aprender a realizar el estudio técnico de los tejidos antiguos y están dirigidos a los especialistas franceses o extranjeros interesados por los problemas de los tejidos antiguos: conservadores, técnicos y restauradores.

Los objetivos de las Secciones consisten en:

- adquirir un método de análisis,

- familiarizarse con los término técnicos definidos en el vocabulario francés del CIETA, y,

- aprender a representar los diversos ligamentos (forma de entrelazarse los hilos de un tejido) por medio de trazados técnicos convencionales.

Estos conocimientos adquiridos a través de los cursos teóricos-prácticos en los que se analizan numerosas muestras de tejidos, deben permitir a los participante proseguir solos el estudio de los textiles antiguos.

El primer de los cursos trata de los tejidos lisos y aborda los principios de los tejidos labrados (tejidos decorados con diseños más o menos complejos) en los telares de lizos y en los telares de tiro o de lazos.

El segundo curso está destinado al estudio de los tejidos labrados antiguos. Y se exige haber seguido el primero y haber asimilado los métodos y el vocabulario anteriormente presentado.

\section{ACLARACIÓN}

Debido a un error, el artículo del profesor Felipe Criado La Arqueología del Paisaje como programa de gestión integral del Patrimonio Arqueológico (vid. PH $n^{\circ} \mid 4$ ) fué publicado antes de que viera la luz el texto Hacia un modelo integrado de investigación y gestión del Patrimonio Histórico: La cadena interpretativa como propuesta, cuya edición está prevista en el número del boletín correspondiente al próximo mes de septiembre. Debido a que en el primer artículo aparecían referencias a este segundo, rogamos disculpen las confusiones que ello haya podido provocar. Agradecemos también al autor su comprensión ante esta circunstancia. 